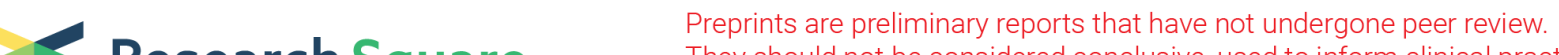 Research Square
or referenced by the media as validated information.
}

\section{Predictors of Pathologic Complete Response in Patients With Residual Flat Mucosal Lesions After Neoadjuvant Chemoradiotherapy for Locally Advanced Rectal Cancer: A Case Control Study}

Chang-Long Li

Peking University Cancer Hospital

Zhen Guan

Peking University Cancer Hospital

Yi Zhao

Peking University Cancer Hospital

Ting-Ting Sun

Peking University Cancer Hospital

Zhong-Wu Li

Peking University Cancer Hospital

Wei-Hu Wang

Peking University Cancer Hospital

Lin Wang

Peking University Cancer Hospital

Ai-Wen Wu ( $\nabla$ drwuaw@sina.com )

Peking University Cancer Hospital

\section{Research Article}

Keywords: Rectal cancer, Preoperative chemoradiotherapy, Tumor regression grade, Flat Mucosal Lesions, Pathologic Complete Response

Posted Date: July 9th, 2021

DOl: https://doi.org/10.21203/rs.3.rs-661023/v1

License: (1) This work is licensed under a Creative Commons Attribution 4.0 International License. Read Full License 


\section{Abstract \\ Background:}

Accurate prediction of tumor response to neoadjuvant chemoradiotherapy (nCRT) remains challenging. There are few studies on pathologic complete response (ypCR) prediction in patients with residual flat mucosal lesions after treatment. This study aimed to identify variables for predicting the ypCR in patients with residual flat mucosal lesions after nCRT for locally advanced rectal cancer (LARC).

\section{Methods:}

Patients with residual flat mucosal lesions after nCRT who underwent radical resection between 2009 and 2015 were retrospectively collected through the LARC database at Peking University Cancer Hospital. Univariate and multivariate analyses of the association between clinicopathological factors and ypCR were performed, and a nomogram was constructed by incorporating the significant predictors.

\section{Results:}

Out of the 246 patients with residual flat mucosal lesions that were included in the final analysis, 56 (22.8\%) had ypCR. Univariate and multivariate analyses showed that posttreatment serum carcinoembryonic antigen (post-nCRT CEA) $\leq 5 \mathrm{ng} / \mathrm{ml}$, magnetic resonance-tumor regression grade (MRTRG) 1 to 3 , and residual mucosal lesion depth $=0 \mathrm{~mm}$ were significantly associated with a higher rate of ypCR. A nomogram was developed with a C-index of 0.735 , and the calibration curve showed that the nomogram model had good predictive consistency.

\section{Conclusion:}

Post-nCRT CEA $\leq 5 \mathrm{ng} / \mathrm{ml}, \mathrm{MR}-\mathrm{TRG} 1$ to 3 , and residual mucosal lesion depth $=0 \mathrm{~mm}$ were predictive factors for ypCR in LARC patients with residual flat mucosal lesions after nCRT. We believe that mucosal re-evaluation before surgery is important as it may contribute to decision-making and facilitating nonoperative management or organ preservation.

\section{Background:}

Rectal cancer is one of the most common tumors worldwide. Due to its insidious onset, most patients are diagnosed with locally advanced rectal cancer (LARC). Neoadjuvant chemoradiotherapy (nCRT) followed by total mesorectal excision (TME) has become the standard treatment for LARC. However,radical surgery still has many disadvantages, including postoperative complications (such as abdominal infection or anastomotic leakage) and dysfunction (such as urination, sexual dysfunction $\square$ or anterior resection syndrome). According to the Consensus on the Watch and Wait Policy in Rectal Cancer Patients After 
Neoadjuvant Treatment (2020 Version), the Watch and Wait strategy (W\&W) or transanal local resection in patients with suspected clinical complete response (CCR) or near clinical complete response (near-cCR) after nCRT could alleviate the pain of surgery and avoid a series of problems caused by organ resection [1]. Pathological response is an important prognostic factor for LARC. Studies have shown that approximately $10 \%-33 \%$ of patients with LARC achieve pathological complete response (ypCR) after nCRT, while up to $70 \%$ patients demonstrate only partial response to treatment [2-6]. The use of the existing diagnosis and treatment methods, such as evaluation of clinical characteristics, imaging changes, and gross morphology of tumors, to improve the prediction of ypCR is of great significance. Although there have been previous studies on the predictors of ypCR in patients after nCRT, the conclusions were diverse and inconsistent. However, there are few studies on ypCR prediction in patients with residual flat mucosal lesions after treatment. Despite the fact that these patients exhibit a good tumor response and large degree of tumor regression, it is difficult to define their CCR or ypCR; This study retrospectively analyzed the predictive factors for $y p C R$ in patients with flat mucosal lesions after nCRT for LARC to determine the optimal treatment for such patients.

\section{Materials And Methods:}

\section{Patient selection}

A total of 246 patients with residual flat mucosal lesions after nCRT and radical resection between 2009 and 2015 were retrospectively collected through the rectal cancer database at Peking University Cancer Hospital. Each patient enrolled in our study satisfied the following criteria: 1) age > 18 years; 2) pathologically proven rectal adenocarcinomas; 3 ) rectal tumors located $<15 \mathrm{~cm}$ from the anal verge; 4) clinically staged as cT3-4 and/or N + rectal tumors; 5) patients treated with nCRT and radical surgery; and 6) patients with residual flat mucosal lesions after nCRT. Patients were excluded if they had 1) distant metastasis or recurrent disease; 2) previous chemotherapy or pelvic radiotherapy. The study was approved by the medical ethics committee of Peking University Cancer Hospital,and waived the informed consent(2021YJZ20).

\section{Neoadjuvant and Surgical Treatment}

All patients received preoperative long-course radiotherapy (LCRT) with concurrent chemotherapy followed by TME surgery.

\section{Imaging assessment}

Magnetic resonance imaging (MRI) was routinely used to determine the clinical stage of the patient before nCRT. After nCRT, the pelvic MR was reviewed again before surgery to determine the tumor's response to treatment and the magnetic resonance-tumor regression grade (MR-TRG) (Table 1) [7-9], which were determined by two radiologists. 
Table 1

The magnetic resonance tumor regression grade score

\section{Grade Radiological complete response (absence of tumor signal and barely visible treatment 1 related scar)}

Grade Good response (dense fibrosis; no obvious residual tumor, signifying minimal residual 2 disease or no tumor)

Grade Moderate response ( $>50 \%$ fibrosis or mucin and visible intermediate signal) 3

Grade Slight response (little areas of fibrosis or mucin but mostly tumor) 4

Grade No response (intermediate signal intensity, same appearances as original tumor/tumor 5 regrowth)

\section{Criteria for flat mucosal lesions}

After radical surgery, the gross tumor specimens were evaluated by experienced pathologists. The depth of the lesion was recorded by vertical measurement in the deepest part of the lesion. The flat mucosal lesions were defined as tumors that regressed after treatment and remained as superficial ulcers or scars with a depth of less than or equal to $5 \mathrm{~mm}$ (Fig. 1).

\section{Statistical data and clinicopathological data}

Data on covariates of interest were collected including age, sex, height $(H)$, weight $(W)$, body mass index (BMI), pretreatment serum carcinoembryonic antigen (pre-nCRT CEA) and carbohydrate antigen 199 (prenCRT CA199) levels, posttreatment serum carcinoembryonic antigen (post-nCRT CEA) and carbohydrate antigen 199(post-nCRT CA199) levels, tumor distance from the anal verge (tumor distance), time interval between chemoradiotherapy and surgery (time interval), and MR-TRG. The tumor size and residual mucosal lesion depth (lesion depth) were recorded, and the ypCR was finally determined by paraffin pathology.

\section{Statistical analysis}

Statistical analyses were performed using IBM SPSS Statistics 25.0. Categorical variables were assessed using the Chi square $(2 * 2)$ or Fisher's exact test $(2 * C)$, where applicable. A p value $<0.05$ was considered significant. Multivariate analysis was performed using a binary logistic regression model (Forward: LR). $\mathrm{R}$ software (4.0.4) and the rms software package were used to construct the nomogram prediction model. The bootstrap method was used for internal validation of the predictive model of the nomogram. The number of repeated sampling times was 1000. The concordance index (C-index) was used to evaluate the predictive performance of the model. Calibration of the nomogram for predicting ypCR rates was performed by comparing the predicted probability and the actual status after bias correction. 


\section{Results:}

A total of 246 patients with residual flat mucosal lesions were included in the final analysis, and 56 $(22.8 \%)$ of them had ypCR. Patient characteristics and clinicopathological data are shown in Table 2. The mean lesion depth was $0.31 \pm 0.19 \mathrm{~cm}$.

Table 2

Patients characteristics

\begin{tabular}{|ll|}
\hline Characteristic & Results \\
\hline Age(year) & $56.46 \pm 11.49$ \\
\hline Sex & $157(63.8 \%)$ \\
\hline Men & $89(36.2 \%)$ \\
\hline Women & $23.97 \pm 3.31$ \\
\hline BMl $(\mathrm{kg} / \mathrm{m} 2)$ & $4.91 \pm 2.04$ \\
\hline Tumor distance $(\mathrm{cm})$ & $9.45 \pm 20.40$ \\
\hline Pre-nCRT CEA (ng/ml) & $29.16 \pm 55.76$ \\
\hline Pre-nCRT CA199 (u/ml) & $3.56 \pm 6.21$ \\
\hline Post-nCRT CEA (ng/ml) & $18.06 \pm 33.30$ \\
\hline Post-nCRT CA199 (u/ml) & \\
\hline MR-TRG & $1(0.4 \%)$ \\
\hline Grade 1 & $17(6.9 \%)$ \\
\hline Grade 2 & $157(63.8 \%)$ \\
\hline Grade 3 & $69(28.0 \%)$ \\
\hline Grade 4 & $2(0.8 \%)$ \\
\hline Grade 5 & $8.92 \pm 2.41$ \\
\hline Time interval $(\mathrm{w})$ & $2.07 \pm 1.04$ \\
\hline Tumor residual size $(\mathrm{cm})$ & $0.31 \pm 0.19$ \\
\hline Lesion depth(cm) & \\
\hline ypCR & $56(22.8 \%)$ \\
\hline Yes & $190(77.2 \%)$ \\
\hline No & \\
\hline
\end{tabular}




\section{Univariable analysis}

Univariate analysis showed that age, sex, BMI, tumor distance, time interval, pre-nCRT CEA, pre-nCRT CA199, post-nCRT CA199, and tumor maximum diameter were not related to the ypCR.

There was a difference in the rate of ypCR between the post-nCRT CEA $\leq 5 \mathrm{ng} / \mathrm{mL}$ group and the post$\mathrm{nCRT}$ CEA $>5 \mathrm{ng} / \mathrm{mL}$ group $(25.2 \%$ vs. $3.6 \%, \mathrm{p}=0.010)$. Similarly, the incidences of ypCR were significantly higher in the MR-TRG $1-3$ group $(29.7 \%$ vs. $5.6 \%, p=0.000)$ and the lesion depth $=0 \mathrm{~mm}$ group $(51.3 \%$ vs. $17.4 \%, p=0.000)$ (Table 3$)$. 
Table 3

Univariable analysis results

\begin{tabular}{|c|c|c|c|c|c|}
\hline Variable & $\mathbf{N}$ & Non-pCR & PCR & $c^{2}$ & $\mathbf{p}$ \\
\hline Sex & & & & 0.752 & 0.386 \\
\hline Men & 157 & $124(79.0 \%)$ & $33(21.0 \%)$ & & \\
\hline Women & 89 & $66(74.2 \%)$ & $23(25.8 \%)$ & & \\
\hline Age(year) & & & & 0.787 & 0.375 \\
\hline$\leq 60$ & 153 & $121(79.1 \%)$ & $32(20.9 \%)$ & & \\
\hline$₫ 60$ & 93 & $69(74.2 \%)$ & $24(25.8 \%)$ & & \\
\hline BMI (kg/m2) & & & & 2.460 & $0.344^{\mathrm{a}}$ \\
\hline$<18.5$ & 9 & $8(88.9 \%)$ & $1(11.1 \%)$ & & \\
\hline $18.5-23.9$ & 118 & $86(72.9 \%)$ & $32(27.1 \%)$ & & \\
\hline$\geq 24$ & 119 & $96(80.7 \%)$ & $23(19.3 \%)$ & & \\
\hline Tumor distance $(\mathrm{cm})$ & & & & 0.371 & 0.542 \\
\hline$\leq 5$ & 163 & $124(76.1 \%)$ & $39(23.9 \%)$ & & \\
\hline$>5$ & 83 & $66(79.5 \%)$ & $17(20.5 \%)$ & & \\
\hline Pre-nCRT CEA (ng/ml) & & & & 0.512 & 0.474 \\
\hline$\leq 5$ & 157 & $119(75.8 \%)$ & $38(24.2 \%)$ & & \\
\hline$>5$ & 89 & $71(79.8 \%)$ & $18(20.2 \%)$ & & \\
\hline Pre-nCRT CA199 (u/ml) & & & & 2.862 & 0.091 \\
\hline$\leq 37$ & 206 & $155(75.2 \%)$ & $51(24.8 \%)$ & & \\
\hline$>37$ & 40 & $35(87.5 \%)$ & $5(12.5 \%)$ & & \\
\hline Post-nCRT CEA (ng/ml) & & & & 6.620 & 0.010 \\
\hline$\leq 5$ & 218 & $163(74.8 \%)$ & $55(25.2 \%)$ & & \\
\hline$>5$ & 28 & $27(96.4 \%)$ & $1(3.6 \%)$ & & \\
\hline Post-nCRT CA199 (u/ml) & & & & 1.480 & $0.224^{\mathrm{b}}$ \\
\hline$\leq 37$ & 231 & $176(76.2 \%)$ & $55(23.8 \%)$ & & \\
\hline \multicolumn{6}{|l|}{${ }^{\text {a} F i s h e r ' s ~ e x a c t ~ t e s t ~}$} \\
\hline${ }^{\mathrm{b}}$ Continuity Correction & & & & & \\
\hline
\end{tabular}




\begin{tabular}{|lcllll|}
\hline Variable & N & Non-pCR & pCR & $\mathbf{c}^{2}$ & p \\
\hline$>37$ & 15 & $14(93.3 \%)$ & $1(6.7 \%)$ & & \\
\hline Time interval (w) & & & & 2.271 & 0.132 \\
$\leq 8$ & 105 & $86(81.9 \%)$ & $19(18.1 \%)$ & & \\
\hline$>8$ & 141 & $104(73.8 \%)$ & $37(26.2 \%)$ & & \\
\hline Tumor residual size (cm) & & & & 0.148 & 0.700 \\
$>3$ & 30 & $24(80.0 \%)$ & $6(20.0 \%)$ & & \\
\hline$\leq 3$ & 216 & $166(76.9 \%)$ & $50(23.1 \%)$ & & \\
\hline Lesion depth (mm) & & & & 21.438 & 0.000 \\
\hline$\geq 1$ & 207 & $171(82.6 \%)$ & $36(17.4 \%)$ & & \\
\hline =0 & 39 & $19(48.7 \%)$ & $20(51.3 \%)$ & & \\
\hline MR-TRG & & & & 16.658 & 0.000 \\
\hline TRG1-3 & 175 & $123(70.3 \%)$ & $52(29.7 \%)$ & & \\
\hline TRG4-5 & 71 & $67(94.4 \%)$ & $4(5.6 \%)$ & & \\
\hline aFisher's exact test & & & & & \\
\hline bContinuity Correction & & & & & \\
\hline
\end{tabular}

\section{Multivariate logistic regression analysis}

Considering that age and time interval might affect tumor development, and the $p$ value of pre-nCRT CA199 was close to 0.05 , we decided to include them in the multivariate logistic regression analysis. Multivariate logistic regression analysis showed that post-nCRT CEA $\leq 5 \mathrm{ng} / \mathrm{ml}(p=0.036$, odds ratio [OR] $=9.267,95 \%$ confidence interval [Cl]: $1.161-73.955)$, MR-TRG 1 to $3(p=0.001, O R=6.397,95 \% \mathrm{Cl}$ : 2.159-18.953), and residual mucosal lesion depth $=0 \mathrm{~mm}(p=0.000, \mathrm{OR}=4.608,95 \% \mathrm{Cl}: 2.127-9.981)$ were predictive factors for ypCR in LARC patients with residual flat mucosal lesions after nCRT (Table 4). 
Table 4

Multivariate logistic regression analysis results

\begin{tabular}{|llll|}
\hline Variable & Odds Ratio & $\mathbf{9 5 \%} \mathrm{Cl}$ & $\mathbf{p}$ \\
\hline post-nCRT CEA & & & \\
$\leq 5 \mathrm{ng} / \mathrm{mL}$ & 9.267 & $1.161-73.955$ & 0.036 \\
\hline$>5 \mathrm{ng} / \mathrm{mL}$ & 1 & & \\
\hline lesion depth & & & \\
\hline Omm & 4.608 & $2.127-9.981$ & 0.000 \\
\hline$\geq 1 \mathrm{~mm}$ & 1 & & \\
\hline MR- TRG & & & \\
\hline TRG1-3 & 6.397 & $2.159-18.953$ & 0.001 \\
\hline TRG4-5 & 1 & & \\
\hline
\end{tabular}

\section{Establishment and verification of the nomogram}

A nomogram was constructed by incorporating the significant predictors identified by multivariate logistic regression, as shown in Fig. 2. Each subtype within these variables was assigned a score on the point scale, which were then added to obtain the total score and probability of ypCR. The original concordance index (orig_c-index) was 0.742 , and the bias-corrected concordance index (bias_corrected_c_index) was 0.735. The results showed that the discrimination ability of the nomogram model was good. In addition, the calibration curve demonstrated that the nomogram model had good predictive consistency (Fig. 3).

\section{Discussion:}

Tumor response to $\mathrm{nCRT}$ is considered to be one of the important factors predicting the prognosis and influencing treatment strategy decisions. However, accurate prediction of the tumor response is still difficult to achieve. Consequently, ypCR patients continue to undergo TME surgery because the tumor response could not be accurately determined before tumor removal. For patients with low rectal cancer who need a definitive abdominoperineal (APR) procedure, the subsequent permanent stoma greatly decreases the quality of life. Increasing experience with the W\&W strategy for CCR or near-cCR through several international registries or single center reports has stressed the importance of judging the status of tumor shrinkage before deciding the surgical plan. This retrospective study was a large sample study exploring the relationship between mucosal changes after nCRT and ypCR. It investigated the prediction of ypCR in patients with residual flat mucosal lesions after nCRT based on several classical factors, and established a nomogram with a maximum prediction efficiency of $60 \%$.

\section{Mucosal appearance of residual disease and ypCR:}


The appearance of post-treatment residual mucosal lesions is important for assessing the treatment response. It is generally believed that the typical endoscopic signs of CCR after chemoradiation therapy include the disappearance of the tumor with healing of the mucosa, decrease in size and complete normalization of the tumor bed, or residual red or white scarring with telangiectasia but without palpable abnormalities [10-12]. However, the reliability of these signs is not very satisfactory, and consistency between cCR and ypCR has not been clarified yet.

Among the 246 patients in our study, 207 patients had incompletely flat lesions (lesion depth $>0 \mathrm{~mm}$ ), of which 36 (17.4\%) were ypCR. However, among the 39 patients with completely flat mucosa (lesion depth $=0 \mathrm{~mm}$ ), the ypCR rate was as high as $51.3 \%$. The results showed that a lesion depth $=0 \mathrm{~mm}$ was associated with ypCR. This suggests the possibility of assessing the post-treatment tumor response by digital rectal exam (DRE) and endoscopy, and if the residual lesions are found to be almost completely flat, these patients could be managed by the W\&W strategy. However, there are some other studies that are worth noting. Fraser et al. and Jemma Bhoday et al. showed that most patients with ypT0 or pCR status did not exhibit mucosal complete clinical response[8, 13]. Therefore, we believe that it may be necessary to combine other parameters to improve the accuracy of ypCR prediction. Consequently, in our study, we explored the synergistic effect of lesion depth, MR-TRG, and post-nCRT CEA for predicting ypCR. The results revealed that the ypCR rate could reach $54.5 \%$ (in lesions with depth $=0 \mathrm{~mm}$ and MR-TRG 1 to 3 ) and $55.6 \%$ (in lesions with depth $=0 \mathrm{~mm}$ and post-nCRT CEA $\leq 5 \mathrm{ng} / \mathrm{ml}$ ) by utilizing the combination of two variables.

\section{MR-TRG and ypCR:}

Our results also showed that MR-TRG 1 to 3 could be an effective indicator for ypCR. We merged MR-TRG $1 / 2 / 3$ together as a good response to make it more practical for clinical application similar to other published data. The MR-TRG stage was chosen as the observed variable rather than MR-T staging because although MRI can provide additional details of the extensive pelvic anatomy compared to ERUS or endoscopy, its accuracy for ypT staging after neoadjuvant therapy is low. The sensitivity of MR for the assessment of post-treatment ypT staging was less than $50 \%$, while the sensitivity for the evaluation of ypT0 was only $19.1 \%[14,15]$.

Similar to our study, Patel et al. reported that good MR-TRG (grade 1 to 3 ) was significantly associated with a favorable pathology [16]. Battersby et al. also suggested that MR-TRG appears to be the most encouraging method for assessing the tumor response to neoadjuvant therapy, and MR-TRG could be used as a key criterion to assess the complete response on the basis of frequent follow-up [17]. Our results are consistent with the above studies. Thus, in patients with lesion depth $=0 \mathrm{~mm}$, we may use MRTRG more convincingly to find the candidates suitable for the W\&W strategy.

\section{Serum CEA and ypCR:}

Previous studies have shown that serum CEA levels might predict the response to treatment and clinical outcome, while the preoperative CEA level was a strong predictor of decreased overall survival and 
systemic recurrence [18-20]. Whether serum CEA could predict ypCR after nCRT is also worth investigating. In our study, multivariate logistic regression analysis showed that a low level of post-nCRT CEA was associated with a higher incidence of ypCR. Our results were consistent with those of other studies by Kleiman et al. and Peng $\mathrm{J}$ et al $[21,22]$. We believe that the reason why pre-nCRT CEA had no association with ypCR was that it was obtained before treatment. Hence, it predominantly reflected the tumor's biologic status rather than its response to nCRT, which is in agreement with Yoon DAE Han's view [23].

However, there is no uniform CEA cut-off value to predict ypCR. Peng J et al. used post-nCRT CEA of $\leq 2$ $\mathrm{ng} / \mathrm{mL}$ as the cut-off value, and they claimed that low post-nCRT CEA levels were associated with ypCR [22]. Moureau-Zabotto et al., Das et al., and Steinhagen et al. reported similar findings with a cut-off of 5 $\mathrm{ng} / \mathrm{mL}$ or $2.5 \mathrm{ng} / \mathrm{mL}[24-26]$. The CEA cut-off value defined by Peng et al. was $5.33 \mathrm{ng} / \mathrm{mL}$, and that by Sun et al. was $5 \mathrm{ng} / \mathrm{mL}[27,28]$. In our study, we set the CEA cut-off value as $5 \mathrm{ng} / \mathrm{ml}$ because it is the maximum threshold of the normal value, which is more in line with diagnostic protocols.

\section{Time interval between chemoradiotherapy and surgery and ypCR:}

Studies showed that prolonging the time interval could increase the ypCR rate [29-31]. However, most of the studies were retrospective studies, consequently, this result should be treated with caution. A multicenter randomized, controlled trial (GRECCAR-6) from France reported that there was no difference in the ypCR rate between the 7-week and 11-week groups[32]. Another prospective study found similar results [33]. In our study, there was no statistical difference in the ypCR rate between the two groups ( $\leq 8$ weeks group and $>8$ weeks group). When we changed the cut-off value to 12 weeks, the ypCR rate of patients in the $>12$ weeks group was higher, but there was no statistical difference. To date, the optimum interval between nCRT and surgery for LARC, in fact, remains controversial. Prospective, randomized, multicenter trials with larger samples are required to draw definitive conclusions.

\section{Limitations:}

Our study has some limitations. Firstly,since all the included patients had flat lesions $\square$ this selection bias might have excluded some patients with a poor tumor response, which might have affected the comparison of ypCR rates. We believe that if all patients were included in the analysis, the final results would be more comparative. Secondly, lymph node metastasis was not considered in this study. However, studies have shown that $\mathrm{N}$ staging is not associated with ypCR[23, 31]. In addition, clinical lymph node staging cannot confirm pathological lymph node staging[34]. Lastly, evaluation and measurement of the lesions using fixed specimens may not be totally consistent with the fresh specimens, and without visual details when compared with endosopic features.

\section{Future Perspective:}


Despite these limitations, the present study consolidated the importance of mucosal changes after nCRT, especially the ypCR predicting value of the combination of residual mucosal lesion depth, MR-TRG, and post-nCRT CEA levels. The nomogram we built might have limited value in patients with different baseline stages or treatment schedules, however, it still reflects the significant factors influencing ypCR rate for reference. In the watch and wait era, our data may further support routine endoscopic re-evaluation before making a surgical plan.

\section{Conclusion:}

Patients with residual flat mucosal lesions after nCRT for LARC should be treated with caution when making treatment strategies. Post-nCRT CEA $\leq 5 \mathrm{ng} / \mathrm{ml}, \mathrm{MR}-\mathrm{TRG} 1$ to 3 , and residual mucosal lesion depth $=0 \mathrm{~mm}$ are predictive factors for $\mathrm{ypCR}$ in LARC patients with residual flat mucosal lesions after nCRT. A nomogram based on these three independent risk factors has a good predictive performance in predicting ypCR. We believe that mucosal re-evaluation before surgery is important and may contribute to decision-making as well as facilitating non-operative management or organ preservation.

\section{Abbreviations}

nCRT

neoadjuvant chemoradiotherapy

ypCR

pathological complete response

LARC

locally advanced rectal cancer

MR-TRG

magnetic resonance-tumor regression grade

TME

total mesorectal excision

W\&W

Watch and Wait strategy

CCR

clinical complete response

near-cCR

near clinical complete response

MRI

Magnetic resonance imaging

pre-nCRT CEA

pretreatment serum carcinoembryonic antigen

post-nCRT CEA

posttreatment serum carcinoembryonic antigen 
pre-nCRT CA199

pretreatment serum carbohydrate antigen199

post-nCRT CA199

posttreatment serum carbohydrate antigen 199

C-index

concordance index

\section{Declarations}

\section{Ethics approval and consent to participate}

The study was approved by the medical ethics committee of Peking University Cancer Hospital, and waived the informed consent(2021YJZ20).

\section{Consent for publication}

Not applicable.

\section{Competing interests}

The authors declared no conflicts of interest.

\section{Acknowledgements}

We would like to thank Editage (www.editage.cn) for English language editing.

\section{Funding}

This study was supported by grants from the National Natural Science Foundation of China (81773214) and the Beijing Municipal Science \& Technology Commission 'Capital Clinical Research Special Fund' (Z151100004015105).

\section{Author information}

Chang-Long Li, Zhen Guan and Yi-Zhao contributed equally as first authors.

\section{Affiliations}


Department of Gastrointestinal Surgery, Key laboratory of Carcinogenesis and Translational Research (Ministry of Education/Beijing), Peking University Cancer Hospital \& Institute, Beijing, PR China.

Chang-Long Li, Yi Zhao, Ting-Ting Sun, Lin Wang \& Ai-Wen Wu

Department of Radiology, Key laboratory of Carcinogenesis and Translational Research (Ministry of Education/Beijing), Peking University Cancer Hospital \& Institute, Beijing, PR China.

Zhen Guan

Department of Pathology, Key laboratory of Carcinogenesis and Translational Research (Ministry of Education/Beijing), Peking University Cancer Hospital \& Institute, Beijing, PR China.

Zhong-Wu Li

Department of Radiation Oncology, Key laboratory of Carcinogenesis and Translational Research (Ministry of Education/Beijing), Peking University Cancer Hospital \& Institute, Beijing, PR China.

Wei-Hu Wang

\section{Authors' contributions}

Chang-Long Li: acquisition of data, analysis \& interpretation of data, drafting the article;

Lin Wang: design \& organizing the study, acquisition of data, analysis \& interpretation of data, revising the article;

Guan Zhen: radiological assessment, acquisition of data;

Yi Zhao: acquisition of data, analysis \& interpretation of data;

Ting-Ting Sun: analysis \& interpretation of data;

Zhong-Wu Li: acquisition of data;

Wei-Hu Wang: acquisition of data;

Ai-Wen Wu: final approval of the article; conception and design of the study, interpretation of the data

\section{Corresponding author}

Dr. Ai-Wen Wu and Dr. Lin Wang are both co-corresponding authors. 


\section{Availability of data and materials}

Original data are available on request by emailing the corresponding author

\section{References}

1. Group(CWWD) CWWDRC: Consensus on the Watch and Wait policy in rectal cancer patients after neoadjuvant treatment (2020 version). Zhonghua Wei Chang Wai Ke Za Zhi 2020, 23(1):1-9.

2. de Campos-Lobato LF, Stocchi L, da Luz Moreira A, Geisler D, Dietz DW, Lavery IC, Fazio VW, Kalady MF: Pathologic complete response after neoadjuvant treatment for rectal cancer decreases distant recurrence and could eradicate local recurrence. Ann Surg Oncol 2011, 18(6):1590-1598.

3. Stipa F, Chessin DB, Shia J, Paty PB, Weiser M, Temple LK, Minsky BD, Wong WD, Guillem JG: A pathologic complete response of rectal cancer to preoperative combined-modality therapy results in improved oncological outcome compared with those who achieve no downstaging on the basis of preoperative endorectal ultrasonography. Ann Surg Oncol2006, 13(8):1047-1053.

4. Park IJ, You YN, Agarwal A, Skibber JM, Rodriguez-Bigas MA, Eng C, Feig BW, Das P, Krishnan S, Crane $\mathrm{CH}$ et al: Neoadjuvant treatment response as an early response indicator for patients with rectal cancer. J Clin Oncol 2012, 30(15):1770-1776.

5. Zorcolo L, Rosman AS, Restivo A, Pisano M, Nigri GR, Fancellu A, Melis M: Complete pathologic response after combined modality treatment for rectal cancer and long-term survival: a metaanalysis. Ann Surg Oncol 2012, 19(9):2822-2832.

6. Xu B, Chen Y, Guo Y, Zhou D, Yue Z, Duan Q, Yang Y, Guan G, Chi P, Lin C: Pretreatment Tumor Thickness as a Predictor of Pathologic Complete Response to Neoadjuvant Chemoradiation Therapy for Stage II/III Rectal Adenocarcinoma. Am J Clin Oncol 2018, 41(6):601-606.

7. Jang JK, Lee JL, Park SH, Park HJ, Park IJ, Kim JH, Choi SH, Kim J, Yu CS, Kim JC: Magnetic resonance tumour regression grade and pathological correlates in patients with rectal cancer. $\mathrm{Br} J$ Surg 2018, 105(12):1671-1679.

8. Bhoday J, Smith F, Siddiqui MR, Balyasnikova S, Swift RI, Perez R, Habr-Gama A, Brown G: Magnetic Resonance Tumor Regression Grade and Residual Mucosal Abnormality as Predictors for Pathological Complete Response in Rectal Cancer Postneoadjuvant Chemoradiotherapy. Dis Colon Rectum 2016, 59(10):925-933.

9. Sclafani F, Brown G, Cunningham D, Wotherspoon A, Mendes LST, Balyasnikova S, Evans J, Peckitt C, Begum R, Tait $D$ et al: Comparison between MRI and pathology in the assessment of tumour regression grade in rectal cancer. British journal of cancer 2017, 117(10):1478-1485.

10. Cho MS, Kim H, Han YD, Hur H, Min BS, Baik SH, Cheon JH, Lim JS, Lee KY, Kim NK: Endoscopy and magnetic resonance imaging-based prediction of ypT stage in patients with rectal cancer who received chemoradiotherapy: Results from a prospective study of 110 patients. Medicine (Baltimore) 2019, 98(35):e16614. 
11. Habr-Gama A, Perez RO, Wynn G, Marks J, Kessler H, Gama-Rodrigues J: Complete clinical response after neoadjuvant chemoradiation therapy for distal rectal cancer: characterization of clinical and endoscopic findings for standardization. Dis Colon Rectum 2010, 53(12):1692-1698.

12. Hupkens BJP, Maas M, Martens MH, van der Sande ME, Lambregts DMJ, Breukink So, Melenhorst J, Houwers JB, Hoff C, Sosef MN et al: Organ Preservation in Rectal Cancer After Chemoradiation: Should We Extend the Observation Period in Patients with a Clinical Near-Complete Response? Ann Surg Oncol 2018, 25(1):197-203.

13. Smith FM, Wiland H, Mace A, Pai RK, Kalady MF: Clinical criteria underestimate complete pathological response in rectal cancer treated with neoadjuvant chemoradiotherapy. Diseases of the Colon and Rectum 2014, 57(3):311-315.

14. Smith N, Brown G: Preoperative staging of rectal cancer. Acta Oncol 2008, 47(1):20-31.

15. van der Paardt MP, Zagers MB, Beets-Tan RGH, Stoker J, Bipat S: Patients Who Undergo Preoperative Chemoradiotherapy for Locally Advanced Rectal Cancer Restaged by Using Diagnostic MR Imaging: A Systematic Review and Meta-Analysis. Radiology 2013, 269(1):101-112.

16. Patel UB, Brown G, Rutten H, West N, Sebag-Montefiore D, Glynne-Jones R, Rullier E, Peeters M, Van Cutsem E, Ricci $S$ et al: Comparison of magnetic resonance imaging and histopathological response to chemoradiotherapy in locally advanced rectal cancer. Ann Surg Oncol 2012, 19(9):2842-2852.

17. Battersby NJ, Moran B, Yu S, Tekkis P, Brown G: MR imaging for rectal cancer: the role in staging the primary and response to neoadjuvant therapy. Expert review of gastroenterology \& hepatology 2014, 8(6):703-719.

18. Tarantino I, Warschkow R, Worni M, Merati-Kashani K, Koberle D, Schmied BM, Muller SA, Steffen T, Cerny $\mathrm{T}$, Guller U: Elevated preoperative CEA is associated with worse survival in stage I-III rectal cancer patients. British journal of cancer 2012, 107(2):266-274.

19. Song S, Hong JC, McDonnell SE, Koong AC, Minsky BD, Chang DT, Liauw SL: Combined modality therapy for rectal cancer: the relative value of posttreatment versus pretreatment CEA as a prognostic marker for disease recurrence. Ann Surg Oncol 2012, 19(8):2471-2476.

20. Park YA, Lee KY, Kim NK, Baik SH, Sohn SK, Cho CW: Prognostic effect of perioperative change of serum carcinoembryonic antigen level: a useful tool for detection of systemic recurrence in rectal cancer. Ann Surg Oncol 2006, 13(5):645-650.

21. Kleiman A, Al-Khamis A, Farsi A, Kezouh A, Vuong T, Gordon PH, Vasilevsky CA, Morin N, Faria J, Ghitulescu G et al: Normalization of CEA Levels Post-Neoadjuvant Therapy is a Strong Predictor of Pathologic Complete Response in Rectal Cancer. J Gastrointest Surg 2015, 19(6):1106-1112.

22. Peng J, Lin J, Wu X, Lu Z, Chen G, Li L, Ding P, Gao Y, Zeng Z, Zhang H et al: Clinical factors of postchemoradiotherapy as valuable indicators for pathological complete response in locally advanced rectal cancer. \#N/A 2016, 71(8):449-454.

23. Han YD, Kim WR, Park SW, Cho MS, Hur H, Min BS, Baik SH, Lee KY, Kim NK: Predictors of Pathologic Complete Response in Rectal Cancer Patients Undergoing Total Mesorectal Excision After Preoperative Chemoradiation. Medicine (Baltimore) 2015, 94(45):e1971. 
24. Moureau-Zabotto L, Farnault B, de Chaisemartin C, Esterni B, Lelong B, Viret F, Giovannini M, Monges $\mathrm{G}$, Delpero JR, Bories E et al: Predictive factors of tumor response after neoadjuvant chemoradiation for locally advanced rectal cancer. Int J Radiat Oncol Biol Phys 2011, 80(2):483-491.

25. Steinhagen E, Shia J, Riedel E, Nash GM, Weiser MR, Temple LK, Paty PB, Guillem JG: Response to neoadjuvant therapy in patients with early age-of-onset rectal cancer. Dis Colon Rectum 2013, 56(1):58-63.

26. Das P, Skibber JM, Rodriguez-Bigas MA, Feig BW, Chang GJ, Wolff RA, Eng C, Krishnan S, Janjan NA, Crane $\mathrm{CH}$ : Predictors of tumor response and downstaging in patients who receive preoperative chemoradiation for rectal cancer. Cancer 2007, 109(9):1750-1755.

27. Peng H, Wang C, Xiao W, Lin X, You K, Dong J, Wang Z, Yu X, Zeng Z, Zhou T et al: Analysis of Clinical characteristics to predict pathologic complete response for patients with locally advanced rectal cancer treated with neoadjuvant chemoradiotherapy. Journal of Cancer 2018, 9(15):26872692.

28. Sun Y, Chi P, Lin H, Lu X, Huang Y, Xu Z, Huang S, Wang X: A nomogram predicting pathological complete response to neoadjuvant chemoradiotherapy for locally advanced rectal cancer: implications for organ preservation strategies. \#N/A 2017, 8(40):67732-67743.

29. Petrelli F, Sgroi G, Sarti E, Barni S: Increasing the Interval Between Neoadjuvant Chemoradiotherapy and Surgery in Rectal Cancer: A Meta-analysis of Published Studies. Ann Surg 2016, 263(3):458-464.

30. Evans J, Bhoday J, Sizer B, Tekkis P, Swift R, Perez R, Tait D, Brown G: Results of a prospective randomised control 6 vs 12 trial: Is greater tumour downstaging observed on post treatment MRI if surgery is delayed to 12-weeks versus 6-weeks after completion of neoadjuvant chemoradiotherapy? Annals of Oncology 2016, 27.

31. Kalady MF, de Campos-Lobato LF, Stocchi L, Geisler DP, Dietz D, Lavery IC, Fazio VW: Predictive factors of pathologic complete response after neoadjuvant chemoradiation for rectal cancer. Ann Surg 2009, 250(4):582-589.

32. Lefevre JH, Mineur L, Kotti S, Rullier E, Rouanet P, de Chaisemartin C, Meunier B, Mehrdad J, Cotte E, Desrame $J$ et al: Effect of Interval (7 or 11 weeks) Between Neoadjuvant Radiochemotherapy and Surgery on Complete Pathologic Response in Rectal Cancer: A Multicenter, Randomized, Controlled Trial (GRECCAR-6). J Clin Oncol 2016, 34(31):3773-3780.

33. Francesca De Felice, Luciano Izzo, Daniela Musio, Anna Lisa Magnante, Nadia Bulzonetti, Federico Pugliese, Paolo Izzo, Pierfrancesco Di Cello, Pietro Lucchetti SI, Tombolini V: Clinical predictive factors of pathologic complete response in locally advanced rectal cancer. Oncotarget 2016, 7(22):33374-33380.

34. Klessen C, Rogalla P, Taupitz M: Local staging of rectal cancer: the current role of MRI. European Radiology 2006, 17(2):379-389.

\section{Figures}



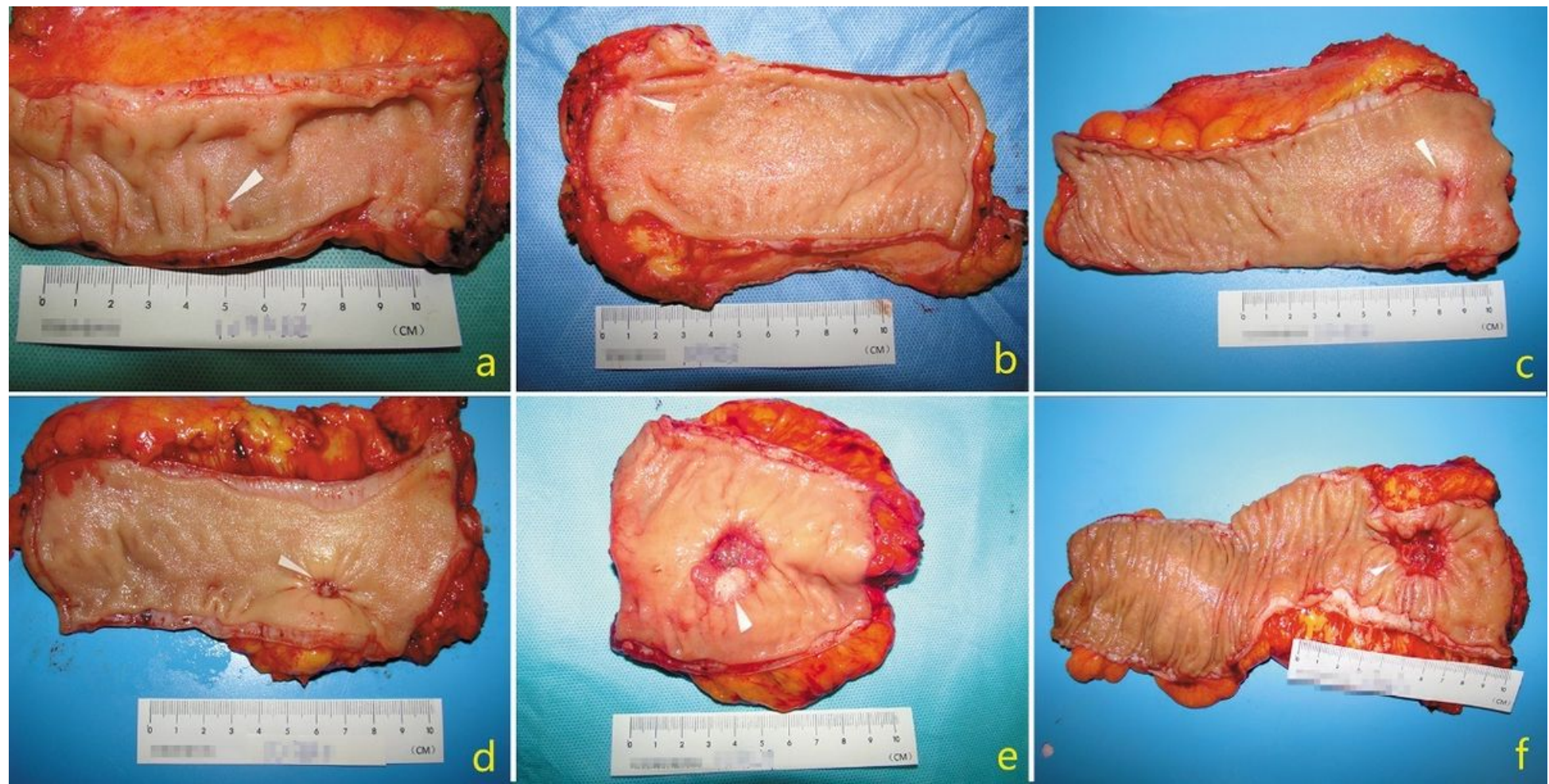

\section{Figure 1}

Typical findings of residual flat mucosal lesions after nCRT on examination of the gross resected specimens: The images a, b, c, d, e, and f show lesions with a depth of $0 \mathrm{~mm}, 1 \mathrm{~mm}, 2 \mathrm{~mm}, 3 \mathrm{~mm}, 4 \mathrm{~mm}$, and $5 \mathrm{~mm}$, respectively 
Points

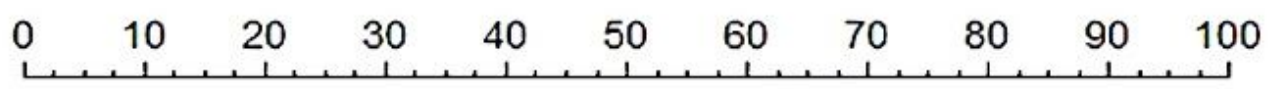

post_CEA

$$
>5 \mathrm{ng} / \mathrm{ml}
$$

lesion_depth

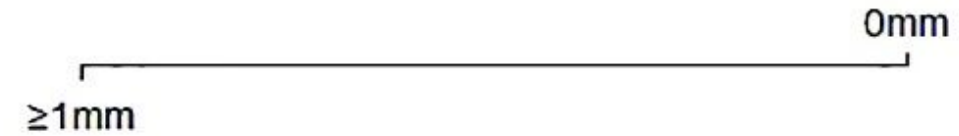

mrTRG

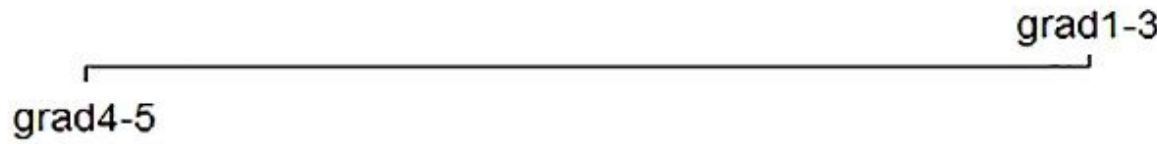

Total Points

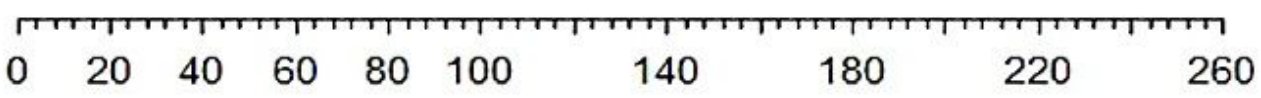

Risk of ypCR

\begin{tabular}{llllllll}
\hline 0.01 & 0.05 & 0.1 & 0.2 & 0.3 & 0.4 & 0.5 & 0.6
\end{tabular}

Figure 2

Nomogram to predict the probability of achieving ypCR 


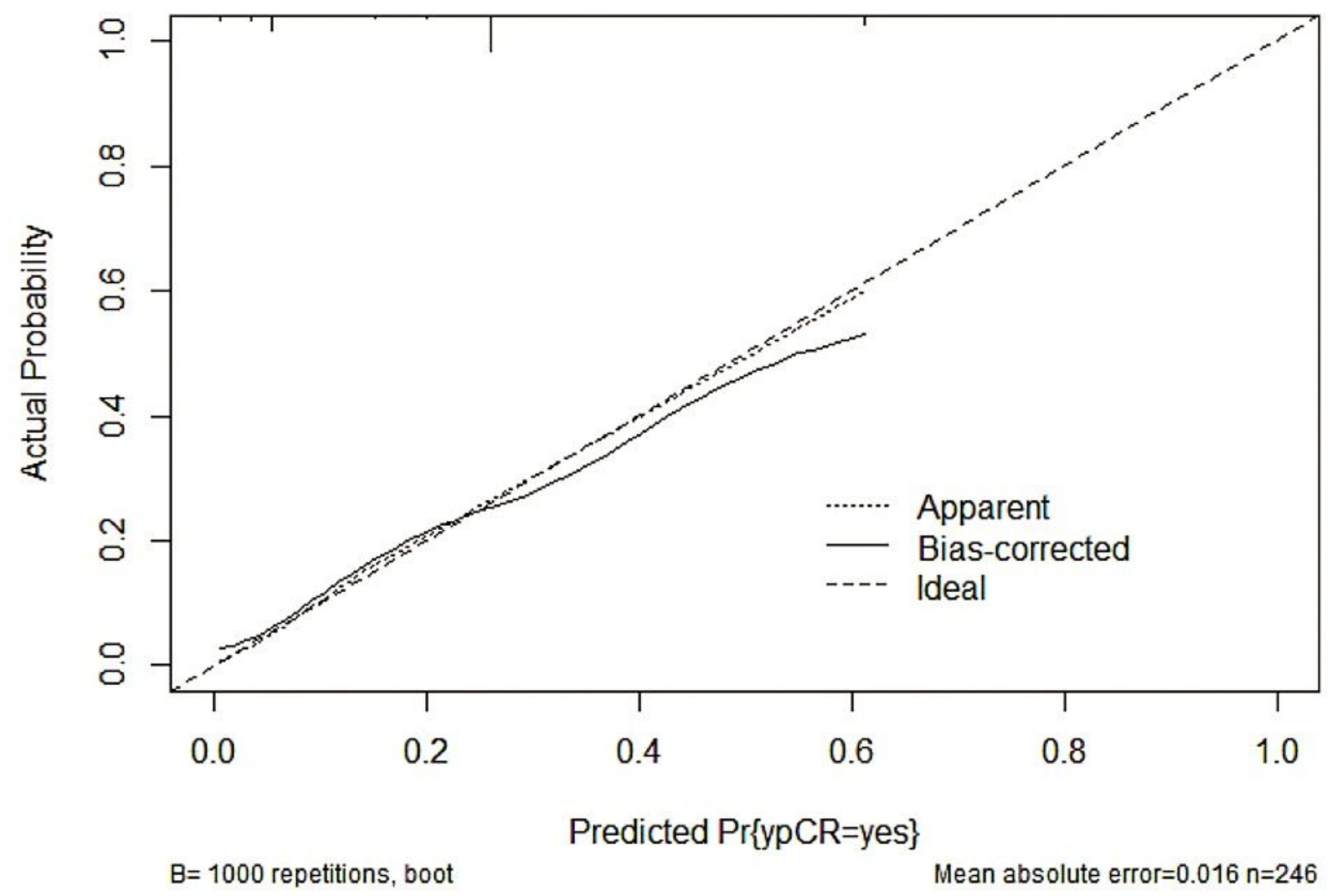

Figure 3

Calibration curve for predicting ypCR 